\title{
Anisotropy on the Fermi surface of the two-dimensional Hubbard model
}

\author{
Y. Otsuka, ${ }^{1, *}$ Y. Morita, ${ }^{1}$ and Y. Hatsugai ${ }^{1,2}$ \\ ${ }^{1}$ Department of Applied Physics, University of Tokyo, 7-3-1 Hongo Bunkyo-ku, Tokyo 113-8656, Japan \\ ${ }^{2}$ PRESTO, Japan Science and Technology Corporation, Kawaguchi-shi, Saitama, 332-0012, Japan
}

(Received 7 May 2002; published 26 August 2002)

\begin{abstract}
We investigate anisotropic charge fluctuations in the two-dimensional Hubbard model at half-filling. By the quantum Monte Carlo method, we calculate a momentum-resolved charge compressibility $\kappa(\boldsymbol{k})$ $=d\langle n(\boldsymbol{k})\rangle / d \mu$, which shows the effects of an infinitesimal doping. At the temperature $T \sim t^{2} / U, \kappa(\boldsymbol{k})$ shows a peak structure at the $( \pm \pi / 2, \pm \pi / 2)$ points along the $\left|k_{x}\right|+\left|k_{y}\right|=\pi$ line. A similar peak structure is reproduced in the mean-field calculation for the $d$-wave pairing state or the staggered flux state.
\end{abstract}

DOI: 10.1103/PhysRevB.66.073109 PACS number(s): 71.10.Fd, 71.10.Pm, 71.27.+a, 79.60.-i

The effects of electron-electron interaction have attracted much nterest. One of the most significant phenomena in a strongly correlated electron system is the Mott transition, which is a quantum phase transition driven by the interaction. This Mott insulator has a finite charge gap, and the anitiferromagnetism often accompanies. Recently, various anomalous properties were found near the Mott transition. There has been a proposal that the interaction brings about anisotropy in the low-energy excitations. For example, the spectral weight of the two-dimensional Hubbard model was investigated. ${ }^{1-4}$ In addition, the deformation of the Fermi surface (FS) due to the interaction occurs in the $t-J$ model. ${ }^{5,6}$ A singular momentum dependence was also observed experimentally. Angle-resolved photoemission spectroscopy measurements suggested anisotropic properties in the low-energy excitations. ${ }^{7}$ These anomalous properties were widely observed in the strongly correlated electron system, and they could be an evidence of the non-Fermi-liquid behavior. It is thus an important and appealing topic to study the lowenergy excitations with momentum resolution.

The anisotropy in low-energy excitations is natural, if we assume a $d$-wave pairing state or a flux state. ${ }^{8}$ They both have a singular energy dispersion that has gap nodes along the diagonal directions $\left|k_{x}\right|=\left|k_{y}\right|$, while a gap opens around the $( \pm \pi, 0)$ and $(0, \pm \pi)$ points. Thus one expects anisotropic charge excitations near the Fermi surface. In general, however, these states compete with other instabilities. Especially at a rational filling, the system often belongs to an antiferromagnetic Mott insulator where the charge degree of freedom is frozen. In this case, the Néel state well describes the ground state. Nevertheless, the $d$-wave pairing state or the flux state can give a sound basis for the interpretation of some singular phenomena near the Mott insulator at a finite temperature.

In this paper, we investigate the charge fluctuation in the half-filled Hubbard model on a two-dimensional square lattice. Using the quantum Monte Carlo method, we calculate the momentum-resolved charge compressibility

$$
\kappa(\boldsymbol{k})=\frac{d\langle n(\boldsymbol{k})\rangle}{d \mu},
$$

where $\langle n(\boldsymbol{k})\rangle=\left\langle c_{k}^{\dagger} c_{k}\right\rangle$ denotes the momentum distribution function and $\mu$ the chemical potential. The integral of $\kappa(\boldsymbol{k})$ is equal to the charge compressibility $\kappa=\int d \boldsymbol{k} \kappa(\boldsymbol{k})$. If the system has a finite charge gap, the charge compressibility decreases exponentially toward zero as the temperature is lowered. Thus, at half-filling, we cannot calculate $\kappa(\boldsymbol{k})$ with sufficient numerical accuracy at a very low temperature. At a finite temperature, an infinitesimal shift of $\mu$ causes an infinitesimal doping. This means that $\kappa(\boldsymbol{k})$ reflects the distribution of infinitesimally doped carriers with a momentum resolution $\delta n(\boldsymbol{k}) \approx \boldsymbol{\kappa}(\boldsymbol{k}) \delta \mu$. Without the interaction, $\boldsymbol{\kappa}(\boldsymbol{k})$ is peaked on the Fermi surface and its value is constant. At half-filling, this non-interacting Fermi surface is a square in the Brillouin zone, $\left|k_{x}\right|+\left|k_{y}\right|=\pi$. We define the Fermi surface as this square in this paper.

We calculate $\boldsymbol{\kappa}(\boldsymbol{k})$ at half-filling. Here the charge degree of freedom is almost frozen and the system is dominated only by an insulating fixed point (antiferromagnetic Mott insulator) at the low temperature $\left(T \ll t^{2} / U\right)$. On the other hand, at a sufficiently high temperature $(T \gg U)$, the Coulomb interaction $U$ is irrelevant. We focus on the intermediate temperature region $\left(T \sim t^{2} / U\right)$, expecting an interaction between charge and spin degree of freedom gives non-trivial feature on $\kappa(\boldsymbol{k})$ even at half-filling.

The Hamiltonian of the two-dimensional Hubbard model is given by

$$
\begin{aligned}
\mathcal{H}= & -t \sum_{\langle i, j\rangle, \sigma}\left(c_{i \sigma}^{\dagger} c_{j \sigma}+c_{j \sigma}^{\dagger} c_{i \sigma}\right) \\
& +U \sum_{i}\left(n_{i \uparrow}-1 / 2\right)\left(n_{i \downarrow}-1 / 2\right)-\mu \sum_{i, \sigma} n_{i \sigma},
\end{aligned}
$$

where $\langle i, j\rangle$ denotes the nearest-neighbor links and $t$ the nearest-neighbor hopping amplitude. The system is on a square lattice and we impose periodic boundary conditions.

In order to obtain approximation-free results, we employ the finite temperature auxiliary field quantum Monte Carlo (QMC) method. ${ }^{9-11}$ In this method, physical observables are evaluated in the grand canonical ensemble. This makes it possible to obtain $\kappa(\boldsymbol{k})$ by a direct sampling in the QMC simulations as

$$
\kappa(\boldsymbol{k})=\beta\left(\left\langle n(\boldsymbol{k}) \sum_{\boldsymbol{k}} n(\boldsymbol{k})\right\rangle-\langle n(\boldsymbol{k})\rangle\left\langle\sum_{\boldsymbol{k}} n(\boldsymbol{k})\right\rangle\right),
$$



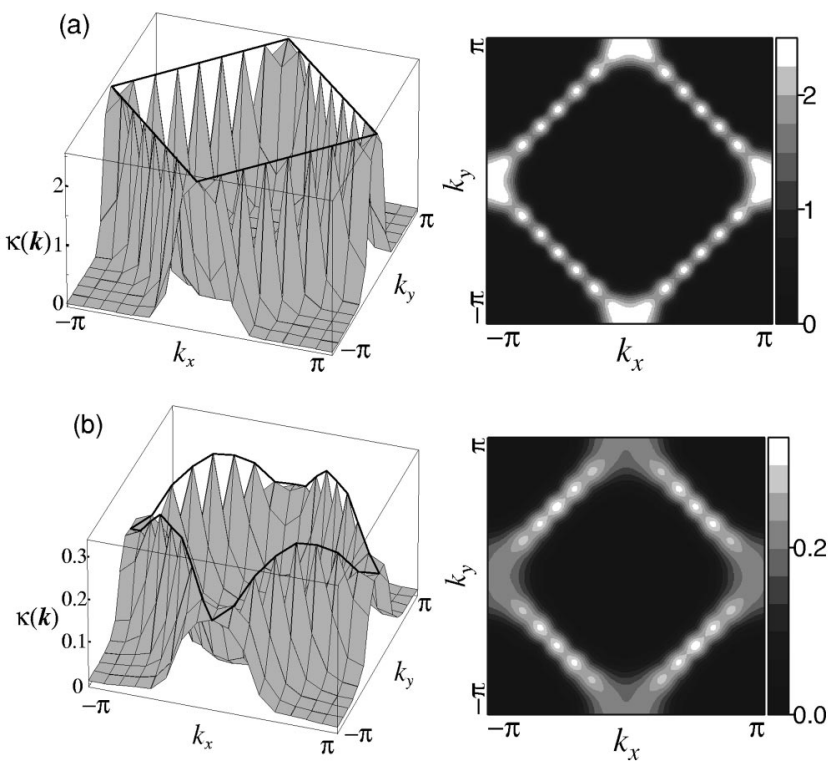

FIG. 1. The momentum-resolved compressibility $\boldsymbol{\kappa}(\boldsymbol{k})$ $=d\langle n(\boldsymbol{k})\rangle / d \mu$ obtained by the quantum Monte Carlo simulations at $T / t=0.2$ on a $16 \times 16$ lattice: (a) for $U / t=0$ and (b) for $U / t=4$. The areas with large values of $\boldsymbol{\kappa}(\boldsymbol{k})$ are highlighted for the contour plots. Without the interaction $(U / t=0), \kappa(\boldsymbol{k})$ is constant on the Fermi surface. On the other hand, for $U / t=4, \kappa(k)$ shows a peak structure at the $( \pm \pi / 2, \pm \pi / 2)$ points.

where $\beta$ denotes an inverse temperature. If we calculate $\kappa(\boldsymbol{k})$ by numerically differentiating the QMC data $\langle n(\boldsymbol{k})\rangle$, we need to perform simulations for the doped system. This brings about a notorious sign problem, which prevents us from obtaining reliable data. On the other hand, a direct evaluation of $\kappa(\boldsymbol{k})$ only needs a simulation at half-filling, where the sign problem does not occur due to the particlehole symmetry. The number of electrons is not fixed at halffilling in the QMC ensembles, even if we set the chemical potential $\mu=0$. Thus, the information on infinitesimally doped systems is statistically taken into account in $\boldsymbol{\kappa}(\boldsymbol{k})$.

The simulations were performed on a $16 \times 16$ square lattice. The finite size effects on $\boldsymbol{\kappa}(\boldsymbol{k})$ are not observed with this lattice size at the temperatures we studied. The Trotter time slice size is set to be $\Delta \tau \simeq 0.1 / t$. We have checked that the systematic error due to the Trotter decomposition does not change qualitative features. For the interacting case, the strength of the interaction is set to be $U / t=4$, where the charge gap is $E_{g} \simeq 0.6 t .{ }^{12}$ We typically performed 500 Monte Carlo sweeps in order to reach a thermal equilibrium followed by $\sim 10^{4}$ measurement sweeps. The measurements are divided into ten blocks, and the statistical error is estimated by the variance among the blocks.

At first, let us discuss results at the temperature $T \sim t^{2} / U$. The results of $\kappa(\boldsymbol{k})$ for $U / t=0$ and $U / t=4$ are shown in Fig. 1. For the noninteracting case, the value of $\kappa(\boldsymbol{k})$ on the FS is constant. On the other hand, for $U / t=4$, a peak structure emerges in $\kappa(k)$ at the $( \pm \pi / 2, \pm \pi / 2)$ points. This indicates that the $( \pm \pi / 2, \pm \pi / 2)$ points are more sensitive to the shift of the chemical potential than $( \pm \pi, 0)$ or $(0, \pm \pi)$. In other words, the system is more compressible at the $( \pm \pi / 2$,

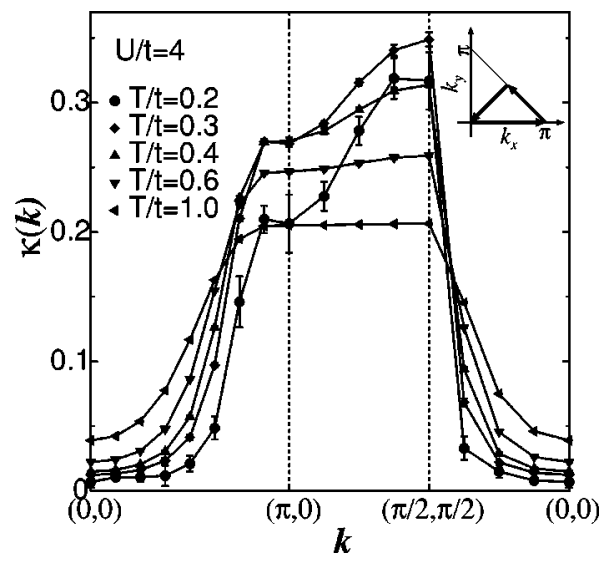

FIG. 2. The momentum-resolved compressibility $\kappa(\boldsymbol{k})$ $=d\langle n(\boldsymbol{k})\rangle / d \mu$ obtained by the quantum Monte Carlo simulations on a $16 \times 16$ lattice for $U / t=4$ at various temperatures. The peak structure at the $( \pm \pi / 2, \pm \pi / 2)$ points vanishes at $T>U$.

$\pm \pi / 2)$ points. Here we note that the interaction does not change the line defined by $\langle n(\boldsymbol{k})\rangle=0.5$, which is identical with the FS in the noninteracting case. In this sense, the shape of the Fermi surface itself is not deformed at half-filling. ${ }^{13}$

Next we discuss the temperature dependence of $\kappa(\boldsymbol{k})$. The results of $\kappa(\boldsymbol{k})$ for $U / t=4$ at several temperatures are provided in Fig. 2. The peak structure at the $( \pm \pi / 2, \pm \pi / 2)$ points is clearly observed at $T \sim t^{2} / U$. It becomes ambiguous as the temperature increases, and vanishes above $T \sim U$. Here we note that the antiferromagnetic correlation length is smaller than the linear system size at these temperatures $(T / t \geqslant 0.2)$. There are two known characteristic energy scales in the Hubbard model. One is the Coulomb interaction $U$ and the other is the effective superexchange interaction $J \sim t^{2} / U$. Our results show that the peak structure in $\kappa(\boldsymbol{k})$ emerges at the temperature $T \sim J$ and vanishes at $T>U$.

In the half-filled Hubbard model, antiferromagnetic longrange order appears at $T=0$. At a finite temperature, the system does not have any long-range order. Then there is no a priori reason to expect such an anisotropy in the charge compressibility. Thus, we compare the QMC results with various mean-field solutions at the temperature $T \sim J$. We focus on three possible mean-field solutions; the Néel state, the $d$-wave pairing state, and the staggered flux state. As an effective model of the Hubbard model, ${ }^{14}$ we use the $t-J$ model to compare with our QMC results,

$$
\begin{aligned}
\mathcal{H}= & -t \sum_{\langle j, k\rangle, \sigma}\left(c_{j \sigma}^{\dagger} c_{k \sigma}+c_{k \sigma}^{\dagger} c_{j \sigma}\right) \\
& +J \sum_{\langle j, k\rangle}\left(\boldsymbol{S}_{j} \cdot \boldsymbol{S}_{k}-\frac{1}{4} n_{j} n_{k}\right)-\mu \sum_{i, \sigma} n_{i \sigma},
\end{aligned}
$$

where $S_{i}=\frac{1}{2} \Sigma_{\sigma, \sigma^{\prime}} c_{i \sigma}^{\dagger} \boldsymbol{\sigma}_{\sigma \sigma^{\prime}} c_{i \sigma^{\prime}}$ and the double occupancy at the same site is prohibited. We take order parameters as 


$$
\begin{gathered}
\Delta_{j k}=\frac{J}{2}\left\langle\left(c_{j \uparrow} c_{k \downarrow}-c_{j \downarrow} c_{k \uparrow}\right)\right\rangle \quad(d \text {-wave pairing), } \\
\chi_{j k}=\frac{J}{2}\left\langle\left(c_{j \uparrow} c_{k \uparrow}^{\dagger}+c_{j \downarrow} c_{k \downarrow}^{\dagger}\right)\right\rangle \quad \text { (staggered flux), } \\
m_{j}=J\left\langle S_{j}^{z}\right\rangle \quad \text { (Néel). }
\end{gathered}
$$

We set $\Delta_{j k}$ to have the $d$-wave symmetry and its amplitude is constant, $\Delta$. The link order $\chi_{j k}$ is chosen so that the effective magnetic flux for each plaquette takes $\phi$ or $-\phi$ alternately. The parameters satisfy $\Delta / t<1$ or $\phi<\pi$ where the low-lying excitations are described by anisotropic Dirac fermions. ${ }^{15}$ The staggered magnetization $m_{j}$ is $(-1)^{j_{x}+j_{y}} m$. In order to apply the mean-field ansatz, we introduce auxiliary fields to decouple the superexchange term and incorporate the constraints of no double occupancy. Next we apply the saddlepoint approximation and neglect the fluctuation around it by taking order parameters as an input parameter (without a self-consistency condition). In low dimensions, the fluctuations generally play a role of destructing the long-range order, and it is natural to expect it in our case. Our focus is, however, not on such a long-distance behavior.

We show $\kappa(\boldsymbol{k})$ at half-filling for these mean-field states in Fig. 3. The peak structure at the $( \pm \pi / 2, \pm \pi / 2)$ points is observed in the $d$-wave pairing or the staggered flux state, which is similar to the QMC results. This implies that the $d$-wave pairing state or the staggered flux state competes with the Néel order at a finite temperature and they give good descriptions of the short-distance behavior. However, we do not claim that these mean-field states become longrange ordered at half-filling. Our interest is in which type of order parameter can give the anisotropy in the charge compressibility at $T \sim J$. Even if these mean-field states are unstable, they can still play an important role at a finite temperature. On the other hand, $\kappa(\boldsymbol{k})$ of the Néel state gives a constant value on the FS, which does not reproduce the $\mathrm{QMC}$ results. The ground state of the half-filled system is well described by the Néel state, while the system behaves essentially as a noninteracting case at a sufficiently high temperature $(T \gg U)$. Both the Néel state and the noninteracting metallic state give a constant $\kappa(\boldsymbol{k})$ on the FS. Therefore, one of the natural scenarios for the anisotropy in $\kappa(\boldsymbol{k})$ is that different kinds of fixed points exist and bring about some singular phenomena at an intermediate temperature. Finally, we note that a possible admixture of different orders may happen at a low temperature.

In summary, we have investigated the charge fluctuation in the two-dimensional Hubbard model by the quantum (a)

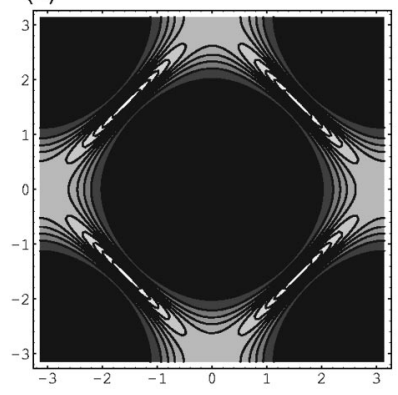

(b)

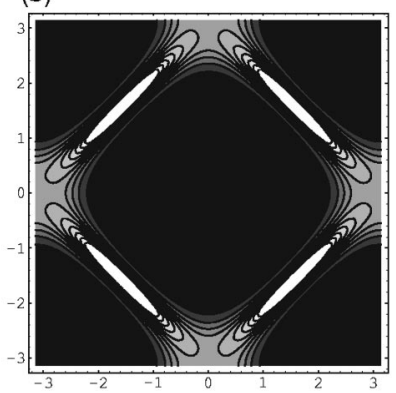

(c)

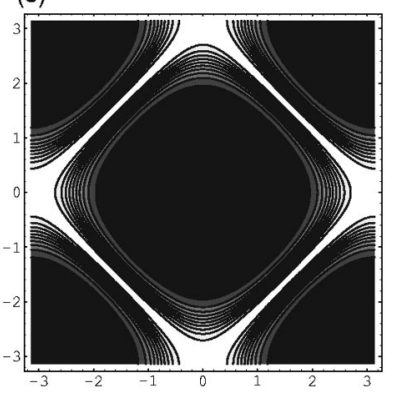

FIG. 3. The mean-field calculations of the momentum-resolved compressibility $\kappa(\boldsymbol{k})=d\langle n(\boldsymbol{k})\rangle / d \mu$ at $T / t=0.2$ : (a) for the $d$-wave pairing state $(\Delta / t=0.2)$, (b) for the staggered flux state $(\phi=\pi / 6)$, and (c) for the Néel state $(m / t=0.6)$. The areas with large values of $\kappa(\boldsymbol{k})$ are highlighted. The $\kappa(\boldsymbol{k})$ shows a peak structure at the $( \pm \pi / 2, \pm \pi / 2)$ points on the Fermi surface for the $d$-wave or staggered flux state, which is consistent with the quantum Monte Carlo simulations. On the other hand, for the Néel state, $\boldsymbol{\kappa}(\boldsymbol{k})$ is constant on the Fermi surface.

Monte Carlo method. The momentum-resolved charge compressibility $\kappa(\boldsymbol{k})$ is focused on at a finite temperature. It gives information on the infinitesimal doping in the Mott insulator. The peak structure at the $( \pm \pi / 2, \pm \pi / 2)$ points is observed in $\kappa(\boldsymbol{k})$ at the temperature $T \sim t^{2} / U$. It is qualitatively consistent with the calculation for the $d$-wave pairing state or the staggered flux state, while the low temperature $\left(T \ll t^{2} / U\right.$ ) behavior is dominated by the antiferromagnetic Mott insulator. This peak structure disappears at the high temperature $T>U$ where the Coulomb interaction is irrelevant. The crossover observed in our results reflects the existence of several fixed points (including unstable ones) in the strongly correlated electron systems.

We thank M. Imada, Y. Kato, S. Ryu, J. Kishine, K. Yonemitsu, and P. A. Lee for fruitful discussions. The computation in this work was done in part using the facilities of the Supercomputer Center, ISSP, University of Tokyo.
*Electronic address: otsuka@pothos.t.u-tokyo.ac.jp

${ }^{1}$ N. Bulut, D.J. Scalapino, and S.R. White, Phys. Rev. Lett. 73, 748 (1994).

${ }^{2}$ N. Bulut, D.J. Scalapino, and S.R. White, Phys. Rev. B 50, 7215 (1994)

${ }^{3}$ R. Preuss, W. Hanke, and W. von der Linden, Phys. Rev. Lett. 75, 1344 (1995).
${ }^{4}$ M. Imada, A. Fujimori, and Y. Tokura, Rev. Mod. Phys. 70, 1039 (1998).

${ }^{5}$ W.O. Putikka, M.U. Luchini, and R.R. Singh, Phys. Rev. Lett. 81, 2966 (1998).

${ }^{6}$ A. Himeda and M. Ogata, Phys. Rev. Lett. 85, 4345 (2000).

${ }^{7}$ F. Ronning, C. Kim, D.L. Feng, D.S. Marshall, A.G. Loeser, L.L. Miller, J.N. Eckstein, I. Bozovic, and Z.-X. Shen, Science 282, 
2067 (1998).

${ }^{8}$ I. Affleck and J.B. Marston, Phys. Rev. B 37, 3774 (1988).

${ }^{9}$ R. Blankenbecler, D.J. Scalapino, and R.L. Sugar, Phys. Rev. D 24, 2278 (1981).

${ }^{10}$ J.E. Hirsch, Phys. Rev. B 31, 4403 (1985).

${ }^{11}$ S.R. White, D.J. Scalapino, R.L. Sugar, E.Y. Loh, J.E. Gubernatis, and R.T. Scalettar, Phys. Rev. B 40, 506 (1989).

${ }^{12}$ N. Furukawa and M. Imada, J. Phys. Soc. Jpn. 61, 3331 (1992).

${ }^{13}$ C.J. Halboth and W. Metzner, Z. Phys. B: Condens. Matter 102, 501 (1997).

${ }^{14}$ A.B. Harris and R.V. Lange, Phys. Rev. 157, 295 (1967).

${ }^{15}$ Y. Morita and Y. Hatsugai, Phys. Rev. Lett. 86, 151 (2001). 